NBI-HE-94-35

July 1994

\title{
Realization of $W_{1+\infty}$ and Virasoro Algebras in Supersymmetric Theories on Four Manifolds
}

\author{
Andrei Johansen円 \\ The Niels Bohr Institute, \\ Blegdamsvej 17, 2100 Copenhagen, Denmark \\ and \\ The St.Petersburg Nuclear Physics Institute, \\ Gatchina, 188350 St.Petersburg, Russian Federation
}

\begin{abstract}
We demonstrate that a supersymmetric theory twisted on a Kähler four manifold $M=\Sigma_{1} \times \Sigma_{2}$, where $\Sigma_{1,2}$ are $2 \mathrm{D}$ Riemann surfaces, possesses a "left-moving" conformal stress tensor on $\Sigma_{1}\left(\Sigma_{2}\right)$ in the BRST cohomology. The central charge of the Virasoro algebra has a purely geometric origin and is proportional to the Euler characteristic of the $\Sigma_{2}\left(\Sigma_{1}\right)$ surface. This structure is shown to be invariant under renormalization group. We also give a representation of the algebra $W_{1+\infty}$ in terms of a free chiral supermultiplet.
\end{abstract}

\footnotetext{
${ }^{1}$ E-mail: ajohansen@nbivax.nbi.dk / johansen@lnpi.spb.su
} 


\section{Introduction}

Recently [1] it has been shown that four dimensional $N=1$ SUSY gauge theories with an appropriate representation of matter can be twisted on a Kähler manifold. A twisted supersymmetric gauge theory possesses a BRST operator which is nilpotent and does not depend on the external metric. If there is no superpotential for the matter supermultiplet then the action of the theory turns out to be BRST exact. Otherwise it is BRST closed. The topological Yang-Mills theory [2] is actually a particular example of such a twisted supersymmetric gauge model with the fields of matter in the adjoint representation of the gauge group. However in the topological Yang-Mills theory [2] and in its twisted $N=1$ version one considers the cohomologies of the different BRST operators 2. An extension of this construction to the supersymmetric theories without gauge interactions is straightforward (we illustrate it in the present paper). We will henceforth generically name the supersymmetric theories twisted on a Kähler manifold heterotic topological theories.

Such a twisting of the $N=1$ supersymmetric models is a four dimensional analogue of a half-twisting of the 2D theories [4]. Witten [4] has shown that under the operation of a half-twist the Landau-Ginzburg model turns out to be left-moving and conformally invariant. This enables us to extract an essential information about the $N=2$ superconformal algebra realized at the infrared fixed point of the LandauGinzburg model. In particular Witten computed the corresponding elliptic genus. This superconformal algebra is realized on the classes of cohomology of one of the supergenerators of the $N=2$ SUSY algebra. Witten also extended his analysis to the case of $(0,2)$ SUSY models coupled to abelian gauge multiplets [5]. In Ref. [6] this construction has been extended to more complicated 2D $N=2$ models where $W_{N}$ algebras are realized in the cohomology of one of the supergenerators.

The space of physical operators (defined as the classes of cohomology of an appropriate BRST operator) in the heterotic theory is in general more complicated than that of the topological theory. This space contains a ground ring of the local operators which are BRST invariant off-shell. This is a purely topological part of the model and this ring coincides with the ring of local physical operators in Witten's topological Yang-Mills theory provided that the matter is in the adjoint representation of the gauge group. Moreover there exist also operators in the cohomology of the BRST operator which can depend on the external metric and commute with the BRST operator only up to the equations of motion. This structure of the space of physical operators is similar to that of 2D half-twisted models [4, 5. In general the physical correlators after the insertions of such operators depend on the external metric. This is due to the presence of such a metric in the inserted operators. They can also depend on the holomorphic coordinates of the Kähler manifold but not on the anti-holomorphic ones [1]. In the theory without a superpotential the action is BRST exact and, hence, the physical correlators do not depend on the gauge coupling constant. Therefore the path integral for the physical correlators allows for a

\footnotetext{
${ }^{2}$ The formulation of Witten's theory in terms of N=1 twisted SUSY turns out to be very useful for calculations of the Donaldson invariants [3].
} 
localization near the solution of the classical equations of motion.

In the present paper 3 we show that the heterotic topological theory on a four manifold $M=\Sigma_{1} \times \Sigma_{2}$, where $\Sigma_{1,2}$ are 2D Riemann surfaces, possesses two chiral Virasoro algebras in the cohomology of a BRST operator. The central charges of these algebras are invariant under the renormalization group and can be calculated in the weak coupling limit. These central charges turn out to be proportional to the Euler characteristic of the corresponding Riemann surface $\left(\Sigma_{1}\right.$ or $\left.\Sigma_{2}\right)$. One can hope that such a construction can provide us with an important information on the renormalization group in the four dimensional $N=1$ supersymmetric QCD, or it can be useful in the description of string vacua.

We also demonstrate that the $W_{1+\infty}$ [8, 9] algebra can be realized in terms of a free chiral supermultiplet.

The paper is organized as follows. In section 2 we formulate the heterotic topological theory. In section 3 the $W_{1+\infty}$ algebra is realized in terms of a free chiral supermultiplet. In section 4 we consider a realization of a Virasoro algebra in the interacting heterotic topological theories. We close with a brief summary of our results.

\section{Supersymmetric theory on a four dimensional Kähler manifold}

The twisted Lagrangian for the Yang-Mills supermultiplet coupled to a chiral supermultiplet in a representation $R$ of the gauge group reads as follows [1]

$$
\begin{aligned}
L=\frac{1}{e^{2}} \sqrt{g} \operatorname{Tr}\left[F^{\bar{m} \bar{n}} F_{\bar{m} \bar{n}}+i \bar{\lambda}^{m n} D_{m} \chi_{n}-\frac{1}{2} D^{\prime 2}+i g^{\bar{m} m} D^{\prime} F_{\bar{m} m}+i \bar{\lambda} D^{m} \chi_{m}\right]+ \\
+\sqrt{g}\left(\bar{\phi} D^{\bar{m}} D_{\bar{m}} \phi+\bar{\psi}^{\bar{m} \bar{n}} D_{\bar{m}} \psi_{\bar{n}}+\bar{\psi} D^{\bar{m}} \psi_{\bar{m}}+N_{\bar{m} \bar{n}} \bar{N}^{\bar{m} \bar{n}}-\right. \\
\left.\quad-i \bar{\phi} \chi_{n} \psi^{n}-\frac{1}{2} \bar{\psi} \bar{\lambda} \phi+\frac{1}{4} \bar{\psi}_{m n} \bar{\lambda}^{m n} \phi+\frac{1}{2} \bar{\phi} D^{\prime} \phi\right)= \\
=\frac{1}{e^{2}} \sqrt{g}\left\{Q, \operatorname{Tr}\left[-i \bar{\lambda} g^{\bar{m} m} F_{\bar{m} m}-\frac{1}{2} D^{\prime} \bar{\lambda}-\frac{i}{2} \bar{\lambda} \overline{\bar{m} \bar{n}} F^{\bar{m} \bar{n}}\right]+\right. \\
+\sqrt{g}\left\{Q,-\frac{1}{2} \bar{\psi}^{\bar{m} \bar{n}} N_{\bar{m} \bar{n}}+\bar{\phi} D^{\bar{m}} \psi_{\bar{m}}-\frac{1}{2} \bar{\phi} \bar{\lambda} \phi\right\},
\end{aligned}
$$

where $g$ is a determinant of the external Kähler metric $g_{m \bar{m}}, m, n \ldots$ and $\bar{m}, \bar{n} \ldots$ are holomorphic and anti-holomorphic world indices on a Kähler manifold $M$, respectively; $D_{m}$ and $D_{\bar{m}}$ stand for the covariant derivatives in gravitational and gauge fields. The gauge supermultiplet consists of the strength tensor $F_{\mu \nu}$ of the gauge field $A_{\mu}$, the fermionic (gaugino [1]) fields $\chi_{n}, \bar{\lambda}$ and $\bar{\lambda}_{\bar{m} \bar{n}}$ which are the $(1,0),(0,0)$ and $(0,2)$ forms respectively, and the $D^{\prime}$ field which is an auxiliary scalar field. The

\footnotetext{
${ }^{3}$ It is a short version of ref. [7].
} 
chiral supermultiplet includes the scalar fields $\phi$ and $\bar{\phi}$, the fermionic fields $\psi_{\bar{n}}, \bar{\psi}$ and $\bar{\psi}_{m n}$ which are $(0,1),(0,0)$ and $(2,0)$ forms respectively, and the $N_{\bar{m} \bar{n}}$ and $\bar{N}_{m n}$ auxiliary fields. $Q$ is the scalar nilpotent generator of BRST transformations which for the gauge multiplet reads

$$
\begin{gathered}
\delta A_{n}=\chi_{n}, \quad \delta A_{\bar{n}}=0, \quad \delta \chi_{n}=0 \\
\delta \bar{\lambda}=-D^{\prime}, \quad \delta \bar{\lambda}_{\bar{m} \bar{n}}=2 i F_{\bar{m} \bar{n}}, \quad \delta D^{\prime}=0
\end{gathered}
$$

while for the chiral multiplet we have

$$
\begin{gathered}
\delta \phi=0, \quad \delta \psi_{\bar{m}}=D_{\bar{m}} \phi, \quad \delta N_{\bar{m} \bar{n}}=D_{\bar{m}} \psi_{\bar{n}}-D_{\bar{n}} \psi_{\bar{m}}+\frac{1}{2} \bar{\lambda}_{\bar{m} \bar{n}} \phi, \\
\delta \bar{\phi}=\bar{\psi}, \quad \delta \bar{\psi}=0, \quad \delta \bar{\psi}^{\bar{m} \bar{n}}=-2 \bar{N}^{\bar{m} \bar{n}}, \quad \delta \bar{N}^{\bar{m} \bar{n}}=0 .
\end{gathered}
$$

It is easy to see that the generator of these transformations does not depend on the external metric.

One can see that all the fields are combined into different supermultiplets. Indeed in the gauge sector we have the following supermultiplets: $\left(A_{n}, \chi_{n}\right),\left(\bar{\lambda}, D^{\prime}\right)$ and $\left(\bar{\lambda}_{\bar{m} \bar{n}}, F_{\bar{m} \bar{n}}\right)$. In the matter sector the multiplets are given by $\left(\phi, \psi_{\bar{m}}, N_{\bar{m} \bar{n}}\right),(\bar{\phi}, \bar{\psi})$, and $\left(\bar{\psi}_{m n}, \bar{N}_{m n}\right)$.

Fixing the ghost number of the BRST charge to be 1 we have the following dimensions $(d)$ and ghost numbers $(G)$ for the different fields:

$$
\begin{aligned}
& (d, G)\left(A_{n}\right)=(d, G)\left(A_{\bar{n}}\right)=(1,0), \quad(d, G)\left(\chi_{n}\right)=(1,1) \\
& (d, G)(\bar{\lambda})=(d, G)\left(\bar{\lambda}_{\bar{m} \bar{n}}\right)=(2,-1), \quad(d, G)\left(D^{\prime}\right)=(2,0) .
\end{aligned}
$$

and

$$
\begin{gathered}
(d, G)(\phi)=(0,2), \quad(d, G)(\bar{\phi})=(2,-2), \quad(d, G)\left(\psi_{\bar{m}}\right)=(1,1), \quad(2.5) \\
(d, G)(\bar{\psi})=(d, G)\left(\bar{\psi}_{m n}\right)=(2,-1), \quad(d, G)\left(N_{\bar{m} \bar{n}}\right)=(2,0), \quad(d, G)\left(\bar{N}_{m n}\right)=(2,0) .
\end{gathered}
$$

Let us now consider a model of a chiral supermultiplet without gauge interactions. The Lagrangian of a free chiral supermultiplet can be easily read off from eq.(2.1). If there is a nontrivial holomorphic $(2,0)$ form $E_{m n}$ on $M$ (i.e. $\left.H^{2,0}(M) \neq 0\right)$ then it is possible to introduce a superpotential $W(x)$ which induces masses and self-interactions for the quantum fields as follows

$$
\begin{gathered}
L_{i n t}=E^{\bar{m} \bar{n}}\left(\psi_{\bar{m}} \psi_{\bar{n}} W^{\prime \prime}(\phi)+N_{\bar{m} \bar{n}} W^{\prime}(\phi)\right)-S^{m n}\left(\bar{\psi}_{m n} \bar{\psi} W^{\prime \prime}(\bar{\phi})+2 \bar{N}_{m n} W^{\prime}(\bar{\phi})\right)= \\
=E^{\bar{m} \bar{n}}\left(\psi_{\bar{m}} \psi_{\bar{n}} W^{\prime \prime}(\phi)+N_{\bar{m} \bar{n}} W^{\prime}(\phi)\right)+\left\{Q, S^{m n} \bar{\psi}_{m n} W^{\prime}(\bar{\phi})\right\}
\end{gathered}
$$

where $S_{\bar{m} \bar{n}}$ is an arbitrary non-singular $(0,2)$ form on $M$. It is easy to see that

$$
\left\{Q, \int_{M} \sqrt{g} L_{i n t}\right\}=0
$$

and hence the total action of the theory with a superpotential is $Q$-closed (but not $Q$-exact due to the interaction terms). 
The condition of renormalizability for a four dimensional theory implies that $W(x)$ should be a polynomial of a degree not higher than 3 . In the case of the superpotential of a degree 2 a Majorana mass is induced for the chiral supermultiplet, while for the cubic superpotential the $|\phi|^{4}+$ Yukawa interactions are induced. As it is well known [10] the superpotential in SUSY theories is non-renormalizable f. The only renormalization of the action comes from $D$-terms [10]. This fact was very important in the analysis of $2 \mathrm{D} \mathrm{N}=2$ SUSY theories [12, 13, 14, 15, 16, 17, 18, 田, where the $N=2$ superconformal theories were associated to quasihomogeneous superpotentials. In an analogy with the $2 \mathrm{D}$ case in this paper we shall consider the case $W(x)=\lambda x^{N+1} /(N+1)$, where $\lambda$ is a coupling constant, and formally we shall consider all positive integer values of $N$.

We can now define the physical operators as classes of cohomology of the BRST operator $Q$. The local observable for the sector of the gauge multiplet becomes a $(2,0)$ form (of dimension 2) given by

$$
O_{m n}^{(0)}=\operatorname{Tr} \chi_{m} \chi_{n} .
$$

It is to be remarked that the situation here is different from that in ordinary topological theories where the local observables are zero-forms; non-zero forms should usually be integrated over closed cycles to get non-local observables (in the case of the highest forms one gets moduli of the topological theory). The difference here is due to the splitting of four coordinates into holomorphic and anti-holomorphic ones, so that the $(2,0)$ form is effectively a scalar with respect to anti-holomorphic derivatives. We have

$$
\partial_{\bar{k}} \operatorname{Tr} \chi_{m} \chi_{n}=\{Q, \ldots\} .
$$

It follows from this equation that the physical correlators with insertions of this operator are holomorphic with respect to its coordinate. The local operators in the matter sector are given by the gauge invariant functions of the (dimensionless) scalar field $\phi$ which correspond to flat directions of the classical moduli space of vacua [19]. It is also possible to construct non-local operators.

It is worth noticing that we could use the vector field $-V_{\mu}$ for a twisting of the theory. Such a modification of the model corresponds to a change $\epsilon, \eta \rightarrow \eta, \epsilon$. The local operators in this mirror model are antiholomorphic up to BRST exact operators (for example, $\partial_{n} \phi=\{Q, \ldots\}$ ) while their correlators are anti-meromorphic.

In general the twisted supersymmetric theory has an anomaly in the decoupling of the external metric from the path integral. This anomaly originates from the axial anomaly in the twisting fermionic current [1]. There are two contributions: the mixed anomaly which depends on both the gauge field and the gravitational one, and a purely gravitational anomaly. It has been shown in ref. [1] that the mixed anomaly is cancelled if

$$
C_{2}(G)-T(R)=C_{2}(G)-\sum_{i} T\left(R_{i}\right)=0 .
$$

\footnotetext{
${ }^{4}$ Notice however that some subtleties can appear due to infrared effects [1].
} 
Here $R=\sum_{i} R_{i}, R_{i}$ are irreducible representations of the gauge group; $C_{2}(G)$ stands for the Casimir operator and $T\left(R_{i}\right)$ is Dynkin index of irreducible representation $R_{i}$ of the gauge group $G ; \operatorname{Tr}_{A d} t^{a} t^{b}=C_{2}(G) \delta^{a b}$ and $\operatorname{Tr}_{R_{i}} t^{a} t^{b}=T\left(R_{i}\right) \delta^{a b}$, where $\operatorname{Tr}_{A d}$ and $\operatorname{Tr}_{R_{i}}$ are the traces in the adjoint and $R_{i}$ representations of the gauge group respectively; $t^{a}$ and $t^{b}$ stand for generators of the gauge group. The condition of cancellation of the gravitational anomaly reads [1]

$$
\sum_{i} \operatorname{dim} R_{i}-\operatorname{dim} G=0,
$$

where $\operatorname{dim} G$ and $\operatorname{dim} R_{i}$ stand for the dimensions of the adjoint and $R_{i}$ representations of the gauge group respectively. The condition (2.10) has been analysed (for a different problem) in ref. [20]. From that analysis one can easily extract that if the condition (2.10) is fulfilled then

$$
\sum_{i} \operatorname{dim} R_{i}-\operatorname{dim} G \geq 0
$$

The equality in the above equation is satisfied only for the matter in the adjoint representation of the gauge group, i.e. for the twisted $N=2$ Yang-Mills theory [2]. Notice also that the mixed anomaly is absent in the case of a twisted model of chiral supermultiplets without gauge interactions.

We shall assume that the mixed anomaly is cancelled. The purely gravitational anomaly itself does not spoil the BRST invariance of the theory and the notion of the BRST cohomology [0].

The gravitational anomaly for these models has a natural interpretation as a conformal anomaly of the Virasoro algebra which appears in the $Q$-cohomology (see below). Indeed the form of the corresponding term in the effective action can be extracted from the $4 \mathrm{D}$ conformal anomaly in the trace of the energy-momentum tensor (see, e.g. 21]) which in our case reads

$$
<\theta_{\mu}^{\mu}>=(\operatorname{dim} R-\operatorname{dim} G) \frac{1}{3 \cdot 128 \pi^{2}}{ }^{*} R_{\mu \nu \lambda \rho}^{*} R^{\mu \nu \lambda \rho},
$$

where $R_{\mu \nu \lambda \rho}$ is the Riemann tensor. The variation of the effective action $S_{\text {eff }}$ with respect to the metric $g_{\mu \nu}$ reads

$$
\delta S_{\text {eff }}=(\operatorname{dim} R-\operatorname{dim} G) \frac{1}{6 \cdot 128 \pi^{2}} \int_{M} d^{4} x \sqrt{g}^{*} R_{\mu \nu \lambda \rho}^{*} R^{\mu \nu \lambda \rho} \delta \log g .
$$

For the case $M=\Sigma_{1} \times \Sigma_{2}$ with a block diagonal metric

$$
g_{\mu \nu}=\left(\begin{array}{cc}
g_{i j}^{(1)} & 0 \\
0 & g_{k l}^{(2)}
\end{array}\right)
$$

we get for the effective action

$$
S_{\text {eff }}=(\operatorname{dim} R-\operatorname{dim} G) \times\left(\frac{\chi_{1}}{48 \pi^{2}} \int_{\Sigma_{2}} \sqrt{g^{(2)}} R^{(2)} \frac{1}{\Delta^{(2)}} R^{(2)}+\frac{\chi_{2}}{48 \pi^{2}} \int_{\Sigma_{1}} \sqrt{g^{(1)}} R^{(1)} \frac{1}{\Delta^{(1)}} R^{(1)}\right),
$$


where $\chi_{1,2}$ are the Euler characteristics of $\Sigma_{1,2}, R^{(1)}, R^{(2)}$ are the Riemann curvatures of $\Sigma_{1,2}$, and $\Delta^{(1)}, \Delta^{(2)}$ are the corresponding Laplace operators. We observe that this is a sum of two induced Liouville actions for the conformal theories on $\Sigma_{2}$ and $\Sigma_{1}$ with the central charges $\chi_{1}$ and $\chi_{2}$. If the gauge interactions are absent the factor $(\operatorname{dim} R-\operatorname{dim} G)$ changes into $N_{f}$ (the number of chiral supermultiplets).

The effective action (2.15) is non-renormalizable at the multi-loop level. Indeed from the point of view of the supersymmetry this anomaly originates from the anomaly in the axial fermionic current which is used for the twisting of the theory [1]. Since this current has an anomaly that depends only on the classical external gravitational fields it does not have any anomalous dimension and hence the anomaly is non-renormalizable. A different argument can be given which is based on the background superfield formalism for supergravity coupled to matter supermultiplets [22]. Indeed as it has been demonstrated in ref. [22], a superfield diagram for multiloop corrections to an effective action can be represented as an integral over four Grassmann variables with an integrand which is a local expression with respect to the Grassmann coordinates. If we formulate our theory in terms of usual fields (with non-changed spins) the quantum supermultiplets are coupled to a reduced external gravitational superfield that is invariant under one of four supercharges (this supergravity multiplet includes only a veirbein field and a vector one). That means that the integrands in superfield diagrams in such a background do not depend on the Grassmann coordinate corresponding to that supercharge. Therefore such multiloop corrections to the effective action in this special supergravity background vanish due to an integration over Grassmann coordinates. It is straightforward to formulate this conclusion in terms of twisted fields. Thus we see that there are no multiloop corrections to the gravitational anomaly (2.15)and (2.17). This argument is an extension of the usual theorems on non-renormalizability of a superpotential

and of the effective action in the instanton background [23, 24] to the case of a special supergravity background.

\section{Realization of the $W_{1+\infty}$ algebra in terms of a free chiral supermultiplet}

For definiteness we hereby describe the conformal algebra on the $\Sigma_{1}$ Riemann surface. Let for simplicity $\Sigma_{1}=T^{2}$ be a torus with a flat two-dimensional metric. The metric of $M=\Sigma_{1} \times \Sigma_{2}$ can be chosen block diagonal (see eq.(2.16)).

Let us define the following operators

$$
W_{n+1}=2 \pi \int_{\Sigma_{2}} \sqrt{g^{(2)}}\left(-\partial_{1} \bar{\phi} \partial_{1}^{n} \phi+g^{2 \overline{2}} \bar{\psi}_{12} \partial_{1}^{n} \psi_{\overline{2}}\right)
$$

Using the definition of $Q$ in eqs.(2.3) and the equations of motion for a free chiral supermultiplet it is easy to see that these operators are $Q$-closed on mass-shell

$$
\left\{Q, W_{n+1}\right\}=0
$$


Moreover these operators are holomorphic on $\Sigma_{1}$ in cohomology of $Q$

$$
\partial_{\overline{1}} W_{n+1}=2 \pi\left\{Q, \int_{\Sigma_{2}} \sqrt{g^{(2)}}\left(-\partial_{1} \bar{\phi} \partial_{1}^{n} \psi_{\overline{1}}-\partial_{2} \bar{\phi} \partial_{1}^{n} \psi_{\overline{2}}\right)\right\} .
$$

Thus we see that $\partial_{\overline{1}} W_{n+1}$ is $Q$-exact and hence is trivial in cohomology.

It is worth emphasizing that the operators $T=W_{2}$ and $J=W_{1}$ are the 11- and 1- components of $2 \mathrm{D}$ tensors on $\Sigma_{1}$. These are actually the components of the energy momentum tensor and the $U(1)$ current integrated over $\Sigma_{2}$ respectively. This fact is responsible for holomorphicity of $T$ and $J$. Indeed the four dimensional current $J_{\mu}$ that corresponds to an unbroken $U(1) R$-symmetry of the action obeys

$$
\partial_{n} J^{n}+\partial^{n} J_{n}=0
$$

while for the energy-momentum tensor $\theta_{\mu \nu}$ we have

$$
\partial^{n} \theta_{n 1}+\partial^{\bar{n}} \theta_{\bar{n} 1}=0
$$

When integrated over $\Sigma_{2}$ the terms which are the total derivatives in $z^{2}$ and $\bar{z}^{\overline{2}}$ in these equations vanish. In turn it is easy to check that the components $J_{\overline{1}}$ and $\theta_{1 \overline{1}}$ are $Q$-exact because of the $Q$-exactness of the action (for example, $\theta_{1 \overline{1}}$ is a variation of the action with respect to the component $g_{1 \overline{1}}$ of the metric).

We now proceed to show that the operators $W_{n+1}$ generate the $W_{1+\infty}$ algebra in the cohomology of $Q$. To this end let us consider the operator product expansion (OPE) of the operators $W_{n+1}$. We have

$$
\begin{gathered}
W_{n+1}(z, \bar{z}) W_{p+1}(w, \bar{w})=\frac{n ! p !(-1)^{n+1} \chi}{2(z-w)^{n+p+2}}+ \\
+2 \pi \sum_{k=0}^{\infty} \frac{(z-w)^{k}}{k !} \int_{\Sigma_{2}} \sqrt{g^{(2)}} d^{2} v\left[\left(\partial_{w} \bar{\phi} \partial_{w}^{n+k} \phi-\bar{\psi}_{12} \partial_{w}^{n+k} \psi_{\overline{2}}\right) \frac{p !(-1)}{(z-w)^{p+1}}+\right. \\
\left.\left(\partial_{w}^{k+1} \bar{\phi} \partial_{w}^{p} \phi-\partial_{w}^{k} \bar{\psi}_{12} \partial_{w}^{p} \psi_{\overline{2}}\right) \frac{n !(-1)^{n}}{(z-w)^{n+1}}\right]+ \\
+Q-\operatorname{exact} \text { terms }= \\
=\frac{n ! p !(-1)^{n+1} \chi}{2(z-w)^{n+p+2}}+\frac{p+n}{(z-w)^{2}} W_{p+n}(w, \bar{w})+\frac{n}{z-w} \partial_{w} W_{n+p}+f\left(W_{n+p-1}, \ldots, W_{1}\right)+ \\
+ \text { regular terms }+Q-\operatorname{exact} \text { terms }
\end{gathered}
$$

where $\chi=(-1 / 2 \pi) \int_{\Sigma_{2}} \sqrt{g^{(2)}} \partial_{\overline{2}} \partial_{\overline{2}} \rho=2(1-g)$ is the Euler characteristic of the Riemann surface $\Sigma_{2}, z$ and $w$ stand for complex coordinates on the surface $\Sigma_{1}$. Here we used that $\partial_{\bar{n}} \phi=\left\{Q, \psi_{\bar{n}}\right\}, \bar{\psi}=\{Q, \bar{\phi}\}$ and the equations of motion. Thus the OPE for $W_{n+1} W_{p+1}$ is holomorphic on $\Sigma_{1}$ in $Q$-cohomology.

It is easy to see that the operator $T=W_{2}$ generates the holomorphic Virasoro algebra with the central charge $\chi$ which is the Euler characteristic of $\Sigma_{2}$. Moreover 
one can see that the operators $W_{n+1}$ generate the holomorphic $W_{1+\infty}$ algebra. Indeed by introducing the Fourier modes

$$
W_{s}^{n}=\oint \frac{d z}{2 \pi i} z^{n+s} W_{n+1}(z, \bar{z})
$$

we get (in the $Q$-cohomology)

$$
\left[W_{s}^{n}, W_{s^{\prime}}^{p}\right]=\frac{\chi(-1)^{n+1} n ! p !}{2} \cdot \frac{(s+n) \ldots(s-p)}{(n+p+1) !} \delta_{s+s^{\prime}, 0}+\left(s p-n s^{\prime}\right) W_{s+s^{\prime}}^{n+p-1}+R .
$$

Here $R$ stands for terms which depend only on $W_{i}^{k}$ with $k<n+p-1$. The standard $W_{1+\infty}$ commutation relations correspond to $R=0$ [8, 9, 27] while in our algebra $R \neq 0$. However adding to the operators $W_{n+1}$ appropriate linear combinations $\sum_{k=0}^{n-1} a_{k} \partial_{z}^{n-k} W_{k+1}$, where $a_{k}$ are constant coefficients, one can recover the standard commutation relations with $R=0$.

\section{Interacting fields}

Let us now consider a manifold $M=\Sigma_{1} \times \Sigma_{2}$ where the genera of both Riemann surfaces $\Sigma_{1,2}$ are non-zero. In this case the manifold $M$ has $H^{2,0}(M) \neq 0$. Let $E_{m n}$ be a non-trivial holomorphic $(2,0)$ form. For simplicity we shall also assume that $\Sigma_{1}=T^{2}$.

In this case it is possible to introduce a superpotential into our model. Let us consider the model with a quasihomogeneous superpotential $W(x)=\lambda x^{N+1} /(N+1)$, where $\lambda$ is a coupling constant (from now on we shall suppress $\lambda$ for simplicity). In this case the $W_{1+\infty}$ algebra turns out to be broken to a Virasoro one.

Using the equations of motion for the chiral supermultiplet (in the presence of the superpotential) it is easy to check that for any $N$ there is a unique bilinear operator which is $Q$-closed and holomorphic in $Q$-cohomology. It has the following form

$$
T_{N}=W_{2}-\frac{1}{N+1} \partial_{1} W_{1}
$$

so that

$$
\left\{Q, T_{N}\right\}=0, \quad \partial_{\overline{1}} T_{N}=\{Q, \ldots\}
$$

Notice that the 1-component of the $U(1)$ current does not belong to the $Q$-cohomology since the phase symmetry is broken by the superpotential.

The operator $T_{N}$ has a spin 2 and generates the holomorphic Virasoro algebra on $\Sigma_{1}$. In order to check it we calculate the OPE for $T_{N}(z, \bar{z}) T_{N}(w, \bar{w})$. It can be done in the weak coupling limit similarly with the calculation in Ref. [4, 5]. The point is that the superpotential terms in the Lagrangian are dimensionful and make less singular contributions to the OPE as compared to the free ones. In particular it is easy to calculate the central charge which is given by

$$
c_{N}=\chi\left(1-\frac{6}{N+1}+\frac{6}{(N+1)^{2}}\right) \text {. }
$$


The model is renormalizable only if $N=1,2$. For the case $N=1$ which corresponds to the free massive model we have $c_{2}=-\chi / 2$, while for $N=2 c_{3}=-\chi / 3$. In both cases the central charge is non-negative since we assumed that $g>0$.

Let us now discuss the behaviour of this algebra with respect to the renormalization group. The superpotential is not $Q$-exact. Hence the physical correlators can depend on the coupling constant in the superpotential. This is a difference of the present case from the twisted $N=1$ Yang-Mills theory [1] where the whole action is $Q$-exact. However the superpotential is not renormalizable [10 while the $D$-terms which are $Q$-exact are the only renormalizable ones. Thus we conclude that our construction is invariant under the renormalization group. This fact can also be understood as follows. The quantum effects result in only a renormalization of the wave functions by a factor $Z$. In turn the same factor $Z$ appears in the operator $T_{N}$ due to quantum effects. These effects are non-trivial for the operator $T_{N}$ because it is $Q$-closed only on mass-shell. Notice that since the operator $T_{N}$ is an integral of a component of the conserved energy-momentum tensor it does not acquire any its own renormalization factor. After a redefinition of the quantum fields the factor $Z$ disappears in the OPE for $T_{N}$ which is therefore invariant under the renormalization group.

Let us now consider the heterotic topological gauge theory. Let us introduce the following gauge invariant operator

$$
T=2 \pi \int_{\Sigma_{2}} \sqrt{g^{(2)}} d^{2} u\left(\frac{1}{e^{2}}\left[2 g^{2 \overline{2}} F_{12} F_{1 \overline{2}}-i\left(D_{1} \bar{\lambda}\right) \chi_{1}\right]+\left[-\left(D_{1} \bar{\phi}\right)\left(D_{1} \phi\right)+g^{2 \overline{2}} \bar{\psi}_{12}\left(D_{1} \psi_{\overline{2}}\right)\right]\right) .
$$

This operator $T(z, \bar{z})$ obeys

$$
\{Q, T\}=0, \quad \partial_{\overline{1}} T=\{Q, \ldots\} .
$$

Thus the operator algebra generated by $T$ is holomorphic in the $Q$-cohomology $[$. Fixing the gauge in the Lagrangian (2.1) one can calculate the operator product expansion in the weak coupling constant limit because the action of the theory is $Q$ exact. It is easy to check that the operator $T$ indeed generates the Virasoro algebra with the central charge

$$
c=(\operatorname{dim} R-\operatorname{dim} G) \chi .
$$

This value of central charge agrees with the expression for the gravitational anomaly (eq.(2.17)).

\section{Conclusions}

We have shown that in a twisted $N=1$ SUSY model with a single free chiral supermultiplet on the four manifold $M=\Sigma_{1} \times \Sigma_{2}$ there exist two infinite dimensional

\footnotetext{
${ }^{5}$ One could suspect that there exists an anomaly in eq.(4.13) similar to that of ref. [5]. It is easy however to check that this is not the case provided that the mixed anomaly in the effective action gets cancelled [7].
} 
symmetries $W_{1+\infty}$ in the cohomology of the BRST operator. The generators of these algebras are integrals over $\Sigma_{2}\left(\Sigma_{1}\right)$ of the bilinear composite operators. The central charge of the $W_{1+\infty}$ algebra is the Euler characteristic $\chi_{2}\left(\chi_{1}\right)$ of $\Sigma_{2}\left(\Sigma_{1}\right)$. If we switch on a non-trivial quasihomogeneous superpotential these algebras are reduced to the chiral Virasoro algebras with central charges proportional to $\chi_{1,2}$. In the heterotic gauge theory there exist two chiral Virasoro algebras with the central charges $\chi_{1,2}$.

Notice that one can interpret the chiral Virasoro algebra in $Q$-cohomology as an algebra in a $2 \mathrm{D}$ conformal theory on a surface $\Sigma_{1}$ with a classical metric. It is tempting to try to extend such an interpretation allowing the metric on $\Sigma_{1}$ to be a quantum one. In this case we expect to get a $2 \mathrm{D}$ quantum gravity extracted from the $4 \mathrm{D}$ theory.

It is worth noticing that the representation of $W_{1+\infty}$ given here is very close to a representation of this algebra in terms of $2 \mathrm{D}$ free (fermionic or bosonic) fields [25, 26, 27]. It is amusing however that in our representation the central charge has a purely geometric origin.

We also point out that one can try to extend our construction to a model with a multiple number of chiral supermultiplets. In such a model it will be interesting to see if a realization of $W_{N}$ and $W_{\infty}^{p}$ [25] algebras can similarly be obtained directly from a four dimensional field theory.

\section{Acknowledgments}

I am grateful to M.Axenides for interesting discussions of the results of this work and a careful reading of the manuscript. I also thank the high energy group at NBI where this work was finished for its hospitality. The present work was supported in part by a NATO grant GRG 930395.

\section{References}

[1] A.Johansen, Twisting of N=1 SUSY gauge theories and heterotic topological theories. FERMILAB-PUB-93/062-T.

[2] E.Witten. Comm. Math. Phys. 117 (1988) 353.

[3] E.Witten, Supersymmetric Yang-Mills on a four-manifold. Preprint IASSNSHEP-94/5.

[4] E.Witten, On the Landau-Ginzburg description of $N=2$ minimal models. Preprint IASSNS-HEP-93/10.

[5] E.Silverstein and E.Witten. Phys. Lett. B 328 (1994) 307. 
[6] K.Mohri, $N=2$ super $W$ algebra in half-twisted Landau-Ginzburg model. UTHEP-260, hep-th/9307029.

[7] A.Johansen, in preparation.

[8] I.Bakas, Phys. Lett. B 228 (1989) 406; Comm. Math. Phys. 134 (1990) 487.

[9] C.N.Pope, L.J.Romans and X.Shen, Nucl. Phys. B 339 (1990) 191; Phys. Lett. B 242 (1990) 401.

[10] S.J.Gates, Jr., M.T.Grisaru, M.Rocek, and W.Siegel, Superspace, or One Thousand And One Lessons In Supersymmetry (Benjamin-Cummings, 1983).

J.Wess and J.Bagger, Supersymmetry And Supergravity, Princeton University Press (second edition, 1992).

[11] M.A.Shifman and A.I.Vainshtein, Nucl. Phys. B 359 (1991) 571.

[12] E.Martinec, "Criticality, Catastrophes, and Compactifications", in Physics And Mathematics Of Strings, ed. L.Brink, D.Friedan, and A.M.Polyakov (World Scientific, 1990).

[13] C.Vafa and N.Warner, Phys. Lett. 218 B (1989) 51.

[14] W.Lerche, C.Vafa, and N.Warner, Nucl. Phys. B 324 (1989) 427.

[15] P.S.Howe and P.C.West, Phys. Lett. 227 B (1989) 397; Phys. Lett. 244 B (1990) 270.

[16] S.Cecotti, L.Girardello, and A.Pasquinucci, Nucl. Phys. B 238 (1989) 701; Int. J. Mod. Phys. A 6 (1991) 2427.

[17] S.Cecotti, Int. J. Mod. Phys. A6 (1991) 1749; Nucl. Phys. B 355 (1991) 755.

[18] C.Vafa, Mod. Phys. Lett. A6 (1991) 337. 569.

[19] N.Seiberg, Exact Results on the Space of Vacua of Four Dimensional SUSY Gauge Theories. Preprint RU-94-18, hep-th/9402044.

[20] I.G.Koh and S.Rajpoot, Phys. Lett. 135B (1984) 397.

[21] S.M.Christensen and M.J.Duff, Phys. Lett. 76B (1978) 571.

[22] M.T.Grisaru and D.Zanon, Nucl. Phys. B 252 (1985) 578, 591.

[23] M.T.Grisaru, W.Siegel and M.Rocek, Nucl. Phys. B 159 (1979) 429.

[24] V.A.Novikov, M.A.Shifman, A.I.Vainshtein and V.I.Zakharov, Nucl. Phys. B 229 (1983) 381; Phys. Lett. B 166 (1986) 329.

[25] I.Bakas and E.B.Kiristis, Nucl. Phys. B343 (1990) 185; Mod. Phys. Lett. A5 (1990) 2039. 
[26] E.Bergshoeff, C.Pope, L.Romans, E.Sezgin and X.Shen, Phys. Lett. 240 B (1990) 105.

D.A.Depireux, Phys. Lett. 252B (1990) 586.

[27] I.Bakas and E.B.Kiristis. Progress of Theor. Physics, Supplement, 102 (1990) 15. 